\title{
Novel Non-Antibiotic Treatment of Bacterial Infections of the Vagina, A Proof of Concept Study using Single Dose and Triple Dose Regimen
}

\author{
Karin Breding ${ }^{1}$, Ake Lindal ${ }^{2 *}$, David Sagna ${ }^{2}$ and Per-Göran Larsson ${ }^{3}$ \\ ${ }^{1}$ Department of Obstetrics and Gynecology Skaraborgs Hospital Skövde, Sweden \\ ${ }^{2}$ Pharmiva AB, St Lars väg 45, Lund, Sweden \\ ${ }^{3}$ Professor Department of Obstetrics and Gynecology Skaraborgs Hospital Skövde, Sweden and department of IKE University of Linköping, Sweden
}

${ }^{*}$ Corresponding author: Ake Lindal, Pharmiva AB, St Lars väg 45, Lund, Sweden, E-mail: ake@pharmiva.com; al@zelmic.se

Received: 16 Mar, 2018 | Accepted: 17 Apr, 2018 | Published: 25 Apr, 2018

Citation: Breding K, Lindal A, Sagna D, Larsson P-G (2018) Novel NonAntibiotic Treatment of Bacterial Infections of the Vagina, A Proof of Concept Study using Single Dose and Triple Dose Regimen. Gynecol Women's Health Res 1(1): dx.doi.org/10.16966/2689-3096.108

Copyright: (C) 2018 Breding K, et al. This is an open-access article distributed under the terms of the Creative Commons Attribution License, which permits unrestricted use, distribution, and reproduction in any medium, provided the original author and source are credited.

\begin{abstract}
The effect of a novel treatment principle for bacterial vaginosis has been investigated in an open clinical study. 10 patients received a single dose while 20 patients received three applications every third day. The formulation is in the form of vaginal foam, containing lactic acid, hydrogen peroxide and an antimicrobial lipid. The intention of using the foam form for delivery is to avoid the creation of untreated areas, as is the case for other local formulations. Clinical signs, symptom and microbial effects have been studied using strict Amsel criteria and modified Hay/Ison for inclusion and modified Hay/Ison to monitor clinical efficacy pre- and post- menstruation. Therapeutic parameters as $\mathrm{pH}$, malodor, abnormal discharge, itch and pain were monitored during the study. The microbiological was monitored by PCR being able to detect 6 pathogens and Lactobacilli.
\end{abstract}

Results: The microbiological results in both groups indicate that all pathogens are reduced by the treatment while the number of Lactobacilli increases. The therapeutic parameters, $\mathrm{pH}$, malodor and abnormal discharge are instantly improved with lasting effects. The clinical and therapeutic parameters follow the microbial events. All patients experience improvement of symptoms and after a single dose $10 \%$ had the grade "normal" according to Hay/Ison after post-treatment menstruation. The corresponding Figure for three administrations was $30 \%$. The clinical effect of three administrations was similar to 5 days of daily treatments using antimicrobial agents such as metronidazole administered the vaginal route but with superior symptoms relief. No adverse effects related to treatment were registered.

Conclusion: The product had a rapid effect on Hay/Ison grade and produced long-lasting improvements in symptoms, even in patients with relapse of BV. We believe that the results are promising and merit further development to improve treatment of BV and STI.

\section{Introduction}

Bacterial vaginosis (BV) is an infection in which the Lactobacilli that normally populate the vagina are replaced by anaerobic bacteria. Data from the NHANES (National Health and Nutrition Examination Survey) 2001-2004 estimated a $29 \%$ of patient aged 14 to 49 and more than $50 \%$ of African American women were positive to BV based on Nugent criteria [1]. Nonetheless, BV increases the risks of various sexually transmitted infections (STIs), including chlamydia, gonorrhoea and human immunodeficiency virus (HIV) [2-5]. The symptoms of BV including abnormal discharge and malodour expressed as fishy odour which are socially unacceptable and disturbing for the affected women. Moreover, in pregnant women it has been linked to negative outcomes such as preterm birth and miscarriage. [6-8]

The etiology of BV is not fully understood but detailed information of the microbiological state of healthy and infected women has been generated. Composition of the vaginal microbiota in healthy, intermediary and infected women has been described by Shipitsyna and Roos et.al. [9] using quantitative PCR analysis. The relation of $85 \%$ Lactobacilli and $15 \%$ pathogens, G.vaginalis and $A$ vaginae, in healthy women was reversed to $15 \%$ Lactobacilli and $85 \%$ pathogens in infected women. In an intermediary group, a 50/50 relation was found.

The current treatment of BV includes antibiotics, such as clindamycin, and metronidazole/tinidazole, high recurrence if evaluation is done post menstruation. Extended antimicrobial treatment of bacterial vaginosis combined with human Lactobacilli to find the best treatment and minimize the risk of relapses has also been tested. Newly published studies monitoring recurrence using more stringent criteria to define "cured" show a post- menstruation cure rate of less than $30 \%$ for vaginal metronidazole [10-12].

Non-antibiotic treatment for BV includes lactic acid, locally applied gels and probiotic Lactobacilli with cure rates that 
in general are very poor. Dequalinium chlorid in the form of vaginal tables may seem as a better alternative [13] the search for new non-antibiotic candidates illustrates the interest for use other antiseptic and non-antibiotic compounds.

In the present study clinical signs and symptoms and the vaginal flora in patients with bacterial vaginosis using a new product based on a novel approach to local vaginal treatment was investigated in an open-label, single-arm study.

This is a first in man study of this product and our objective has been to study the safety and efficacy of the novel product after a single and a triple dose in women suffering from BV.

The primary objective was to study the effect of hydrogen peroxide with lactic acid in foam to treat BV and the secondary objective was to study the safety of the product used as a singleand multiple-dose vaginal application.

\section{Materials and Methods}

Since the product contain hydrogen peroxide and lactic acid it has a potential for being effective in other diseases as well, such as STI, sexually transmitted infections. The decision to make the first study on bacterial vaginosis is due to the welldeveloped diagnosis for both clinical and microbiological parameters and there is a good understanding on the conditions in healthy and diseased women.

The novel approach based on two known factors regulating the microbiological balance in the vagina, hydrogen peroxide ( 3 $\mathrm{mg} / \mathrm{g})$ and lactic acid $(27 \mathrm{mg} / \mathrm{g})$ both generated by Lactobacilli. The product is presented as vaginal foam and a third compound, a lipid, 1-Glycerol monolaurate, has been added in crystalline form to enhance the foam properties.

Most local applied products are gels, creams or vaginal suppositories and these formulations limit the distribution of the drug. The foam is sprayed though an actuator for vaginal application and fills the entire vaginal cavity and melts just below body temperature providing coverage of the entire vaginal mucus. The product has been tested for antimicrobial effect in vitro demonstrating powerful antimicrobial capacity. In an "In vitro" study the antimicrobial effect on 2 pathogens, $G$. vaginalis and $N$. gonorrhea has been investigated. These species both have the ability to produce catalases, a hydrogen peroxide degrading enzyme. The results indicate that a dilution of 1:1000 of the novel product, containing $0.3 \%$ hydrogen peroxide, is inhibiting growth of Neisseria gonorrhoea ATCC 19424 on Mueller-Hinton agar without sheep blood. With sheep blood the minimum inhibiting concentration is $1: 100$. The results indicate that the novel product can eradicate bacteria independent of the presence of catalases (report on file).

30 patients, age 18 to 49 years with bacterial vaginosis were recruited from the gynecological department at Skaraborg Hospital Skövde. 10 patients were treated by a single dose and 20 by three doses. The first dose was done at the hospital by health care professionals while dose 2 and 3 were administered by the patient at home.
The inclusion criteria were age $\geq 18$ years, regular menstruation cycle, using adequate contraceptive measures and bacterial vaginosis, as defined by fulfilment of at least 3 of the following criteria (Amsel criteria):

a) $\mathrm{pH}$ of vaginal fluid $>4.5$

b) Positive amine test

c) Presence of clue cells

d) Presence of characteristic, homogenous vaginal discharge

The primary efficacy parameters are results generated by modified Hay/Ison method using rehydrated airdried vaginal smears, the grades are as follows: (1) "Normal" - Lactobacilli predominate; (2) "Intermediate" - Mixed flora with Lactobacilli and Gardnerella/Mobiluncus spp. present; and (3) "Bacterial vaginosis" - Predominantly Gardnerella and/or Mobiluncus spp, with few or no Lactobacilli.

Exploratory efficacy variables were patient-reported symptoms of itch, pain, malodour and vaginal discharge, $\mathrm{pH}$ of the vaginal fluid, quantitation of pathogens in relation to Lactobacilli. The quantification of the vaginal flora are done by PCR technique, in the form of a commercial kit delivered by Dynamic Code, Linköping Sweden, allowing quantification of 6 pathogens and total number of Lactobacilli present. The pathogens are Gardnerella vaginalis, Atopobium vaginae, Leptotrichia/Sneathia spp., Megasphaera spp., Mobiluncus spp. and bacterial vaginosis-associated bacterium 2 (BVAB2) all in relation to Lactobacilli. The Lactobacilli count includes all Lactobacilli present.

Exploratory efficacy variables applied were $\mathrm{pH}$ of the vaginal fluid on days 4 and 7 and quantitation of pathogens in relation to Lactobacilli as well on days 4 and 7.

The study comprises three visits. Visit 1 encompassed screening using Amsel criteria, modified Hay/Ison and treatment. After providing informed consent, patients were screened for eligibility. Eligible patients were included in the study and were asked about symptoms of itch, pain, malodour and vaginal discharge. They underwent a gynecological examination during which samples of vaginal fluid were collected by the Principal Investigator. The vaginal fluid samples were used for modified Hay/Ison grading (as "Normal", "Intermediate" or "Bacterial vaginosis") and for typing and quantification of the vaginal flora. Concomitant medications were recorded. The patients were then treated with one dose of the novel product. The patients were asked about their perceptions of the treatment. Adverse event (AE) recording started immediately after treatment. Treatment was not applied during days of menstruation or when menstruation was expected during the first 2 weeks after Visit 1.

\section{First 10 patients}

At visit 1 (day 1), patients were given kits ( $\mathrm{pH}$ and dry smear/PCR test kits) for self-performed vaginal sampling and 
were instructed to use them daily during the first week after treatment. Self-obtained vaginal samples, for modified Hay/ Ison grading and assessment of the vaginal flora as well as recorded $\mathrm{pH}$ values, were sent to the study site every day.

Patients were contacted by telephone after the first week, visit 2. They were asked about AEs, concomitant medications, posttreatment menstruation and symptoms (itch, pain, malodour and vaginal discharge).

One week after the end of the first post-treatment menstruation, the patients also did self- performed vaginal sampling using the $\mathrm{pH}$ and dry smear/PCR test kits.

After the first post-treatment menstruation, the patients received a follow-up clinical consultation, Visit 3, in which they underwent a gynecological examination and were asked about AEs, concomitant medications and symptoms. An overall study design of the first 10 patient is illustrated in Figure 1

\section{Subsequent 20 patients}

The next part of the study was to evaluate three dose regimens. At visit 1 patients were provided with 2 additional doses of the novel product and kits, $\mathrm{pH}$ and dry smear/PCR test kits, for self-performed vaginal sampling. On day 4, 72 hours after the first dose of the novel product, patients did self-performed vaginal sampling at home before administering the second dose of the novel product. On day 7, 144 hours after the first dose of the novel product, patients did self-performed vaginal sampling at home before administering the third dose of the novel product. Patients did self-performed vaginal sampling 1 week after the final dose of the novel product and one week after the first post treatment menstruation. An overall study design of the subsequent 20 patient is illustrated in Figure 2.

The study was conducted in compliance with the protocol, regulatory requirements, good clinical practice (GCP) and the ethical principles of the latest revision of the Declaration of Helsinki as adopted by the World Medical Association. The final study protocol and the final version of the patient

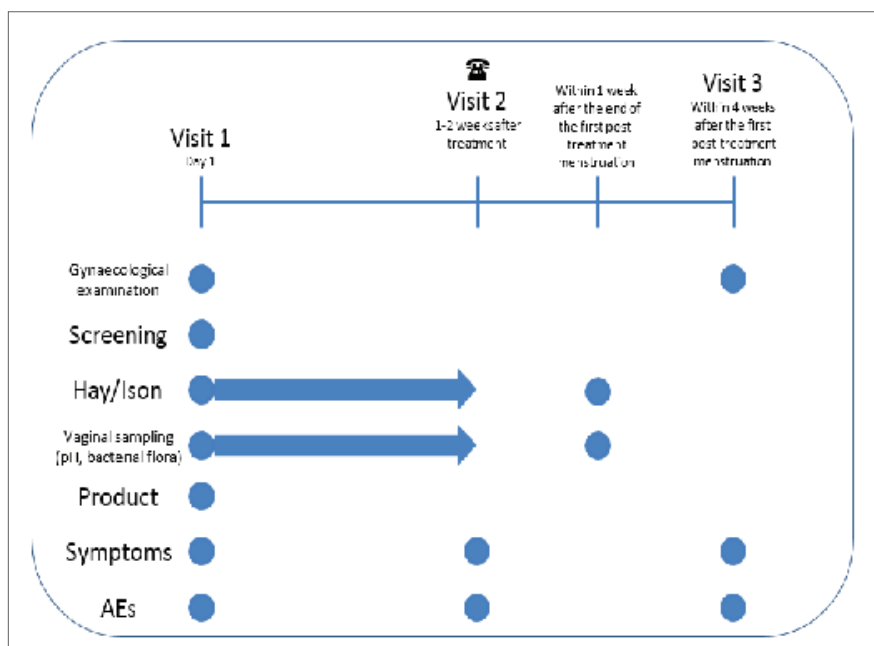

Figure 1: Overall Study Design: First 10 Patients

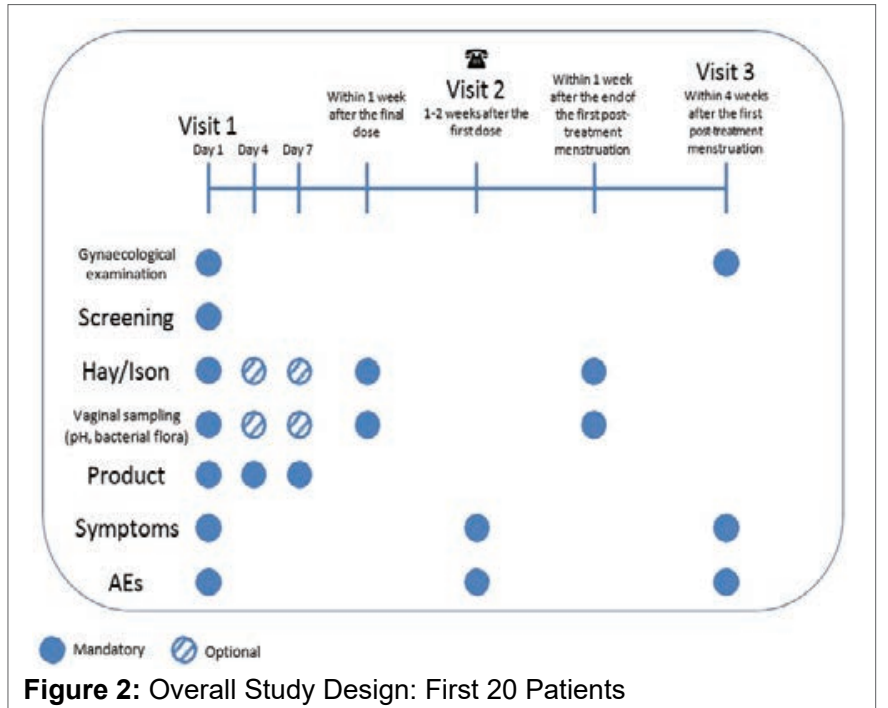

information and consent form were reviewed and approved by an independent ethics committee (IEC) and MPA Sweden prior to inclusion of patients (EudraCT 2015-003356-51).

\section{Statistical methods}

The variability of the measurements associated with the primary objective was unknown when the present study was planned. The number of patients needed was therefore uncertain, but it was judged that 30 patients (fully evaluable) would be enough to meet the primary objective of the study.

\section{Results}

The demographics for the enrolled patients are shown in Table 1. Mean age was 28.6 years (range 18 to 42 ) for the first 10 patients and 31.7 (range 19 to 50) for the subsequent 20 patients.. Eight of the first 10 patients (80\%) and 15 of the subsequent 20 patients $(75 \%)$ had a regular sexual partner. The 2 patients not using contraception at baseline were advised about contraception use.

All of the first 10 patients and 19 of the subsequent 20 patients fulfilled all 4 Amsel criteria at enrolment (Visit 1). The entire enrolled patient (30) was diagnosed with BV according to modified Hay/Ison.

\section{Primary efficacy variable}

Data for the single dos treatment are shown in Table 2. At day 2, all patients had a Hay/Ison grade of "normal". The effect of the single dose languishes and at day 7 one patient was diagnosed "normal", 3 intermediary and 4 had BV. One (10\%) of the patients treated with a single dose (of the novel product) had a Hay/Ison grade of "Normal" 1 week after the end of the first post-treatment menstruation.

For the subsequent 20 patients that received 3 doses of the novel product, $50 \%$ had a Hay/Ison grade of "normal" and 33\% were intermediates after last treatment (day 7). One week after last treatment $47 \%$ were diagnosed "normal" and $12 \%$ were intermediates, see Table 3 . At the end point, 1 week after the 
Table 1: Baseline demographics

\begin{tabular}{|c|c|c|c|}
\hline & & First 10 patients & Subsequent 20 patients \\
\hline \multirow{6}{*}{ Age (years) } & No. of observations & 10 & 20 \\
\hline & Mean & 28.6 & 31.7 \\
\hline & Standard deviation & 8.8 & 10.2 \\
\hline & Median & 26 & 30.5 \\
\hline & Min & 18 & 19 \\
\hline & Max & 42 & 50 \\
\hline \multirow{3}{*}{ Regular menstruation, n (\%) } & Yes & $8(80)$ & $20(100)$ \\
\hline & No & $1(10)$ & $0(0)$ \\
\hline & Missing & $1(10)$ & $0(0)$ \\
\hline \multirow{3}{*}{ Regular sexual partner, n (\%) } & Yes & $8(80)$ & $15(75)$ \\
\hline & No & $2(20)$ & $5(25)$ \\
\hline & Missing & $0(0)$ & $0(0)$ \\
\hline \multirow{6}{*}{ Contraception, n (\%) } & None & $1(10)$ & $2(10)$ \\
\hline & Hormone-based & $6(60)$ & $5(25)$ \\
\hline & Condom & $1(10)$ & $2(10)$ \\
\hline & Intrauterine device & $0(0)$ & $10(50)$ \\
\hline & Pessary & $0(0)$ & $0(0)$ \\
\hline & Other & $2(20)$ & $1(5)$ \\
\hline
\end{tabular}

Table 2: Hay/Ison grade at Visit 1 and during the First Week after Treatment: First 10 Patients

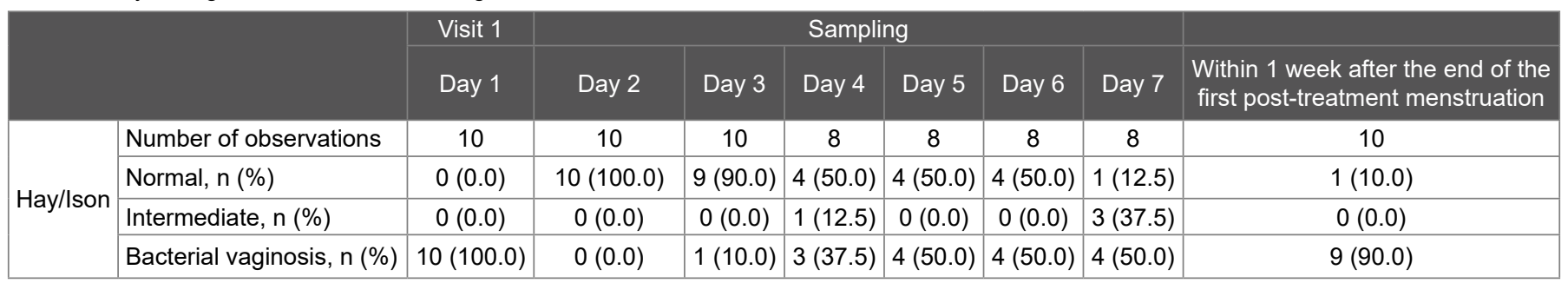

Table 3: Hay/lson grade at Visit 1, Day 4, Day 7 and Within 1 Week after the Final Dose: Subsequent 20 Patients

\begin{tabular}{|c|c|c|c|c|c|c|c|}
\hline & Visit 1 & \multicolumn{2}{|c|}{ Treatment } & \multirow{2}{*}{$\begin{array}{l}\text { Sampling } \\
\text { Within } 1 \\
\text { week after } \\
\text { final dose }\end{array}$} & \multirow{2}{*}{$\begin{array}{l}\text { Sampling } \\
\text { Within } 1 \text { week after } \\
\text { the end of the first } \\
\text { post-treatment } \\
\text { menstruation }\end{array}$} & \multirow{2}{*}{$\begin{array}{c}\text { Visit } 3 \\
\begin{array}{l}\text { Within } 1 \text { month after the end of the } \\
\text { first } \\
\text { post-treatment menstruation }\end{array}\end{array}$} \\
\hline & & Day 1 & Day 4 & Day 7 & & & \\
\hline \multirow{4}{*}{ Hay/lson } & Number of observations & 20 & 18 & 18 & 17 & 17 & 18 \\
\hline & Normal, n (\%) & $0(0.0)$ & $10(55.6)$ & $9(50.0)$ & $8(47.1)$ & $4(23.5)$ & $5(27.8)$ \\
\hline & Intermediate, n (\%) & $0(0.0)$ & $3(16.7)$ & $6(33.3)$ & $2(11.8)$ & $1(5.9) a$ & $0(0.0)$ \\
\hline & Bacterial vaginosis, n (\%) & $20(100.0)$ & $5(27.8)$ & $3(16.7)$ & $7(41.2)$ & $12(70.6)$ & $13(72.2)$ \\
\hline
\end{tabular}

Data shown as number of patients(\%)

a The patient with a Hay/lson grade of "Intermediate" after the end of the first post-treatment menstruation (patient 19) was shown to be cured at Visit 3.

end of the first post-treatment menstruation, 4 of 17 patients were regarded as normal (24\%). One patient (6\%) had a Hay/ Ison grade of "Intermediate". This patient was shown to be cured at Visit 3. Inclusion of additional data for this patient, and for another patient, patient 20 who failed to provide a sample for Hay/Ison grading after the end of the first post-treatment menstruation but was shown at Visit 3 to have $\mathrm{BV}$, gives a cure rate of $27.8 \%$ ( 5 of 18 patients).

\section{Exploratory endpoints Bacteriology}

The sampling for microbiological testing was performed by the patients except for the first sampling occasion which was performed by health care professionals at the clinic. Patient performed sampling has been done before, with good results [14].

All patients were also tested for vaginal fluid microbiological status using PCR-analysis for Gardnerella vaginalis, Atophobium vaginae, Leptotrichia sp, Megasphaera sp, Mobiluncus sp and BVAB2. We have evaluated the presence of these bacteria in relation to the quantity of total Lactobacillus present.

The results of the testing demonstrate that the six pathogens monitored are immediately reduced and that Lactobacilli, including four different species, start to increase in numbers after the first dose. The effect on both pathogens and 
Lactobacilli after a single dose peaks after 2 to 3 days and is back to pretreatment values after 6 to 7 days (Figures 3 and 4)

In the group receiving 3 doses given three days apart, the initial effect seems identical, a rapid decrease of pathogens and an increase of Lactobacilli. However, the maximal effect is maintained for at least one week after last treatment (Figures 5 and 6). In the group receiving a single dose the effect started to decrease after two to three days. This indicates that multiple dosing can generate additional or accumulated effects.

Post hoc exploratory analyses of vaginal pathogens and Lactobacilli were performed for subgroups of patients who received 3 doses of the product. Data for patients with a Hay/ Ison grade of "Normal" or "Intermediate" within 1 week after the end of the first post-treatment menstruation are shown in Figures 7 and 8. The initial decrease in the populations of pathogenic species and increase in total Lactobacilli numbers were maintained throughout the study period in these patients.

\section{Therapeutic efficacy variables}

The change in therapeutic parameters, itch, pain, malodour and vaginal discharge of the vaginal fluid during treatment compared with Visit 1 are shown in Table 4 (first 10 patients) and Table 5 (subsequent 20 patients). For patients who received a single dose of the product (first 10 patients), the 3 patients who reported itch at Visit 1 and 1 patient who reported pain at Visit 1 reported improvements in their symptoms at Visit 3. Compared to Visit 1, malodour was improved in all 10 patients at Visit 2 and in 5 patients (50\%) at Visit 3. Vaginal discharge was improved in $80 \%$ of patients at Visit 2 and in $40 \%$ of patients at Visit 3.

For patients who received 3 doses of the product, malodor improved in 19 patients (100\%) between Visit 1 and Visit 2, and was less severe at Visit 3 vs Visit 1 in 16 patients (94.1\%).

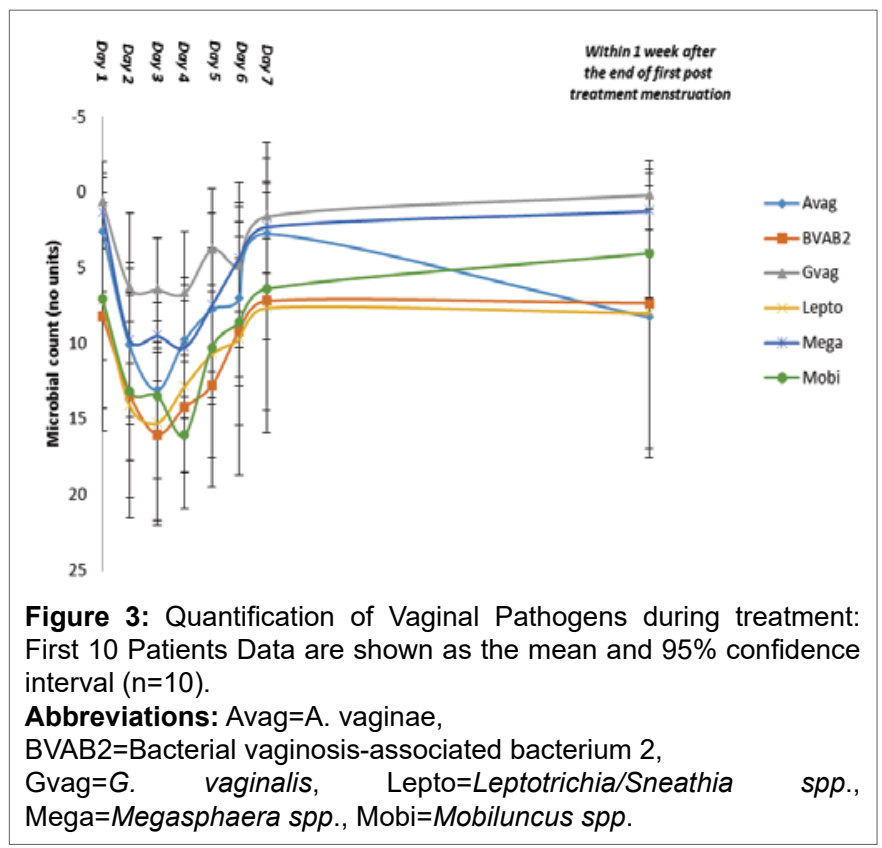

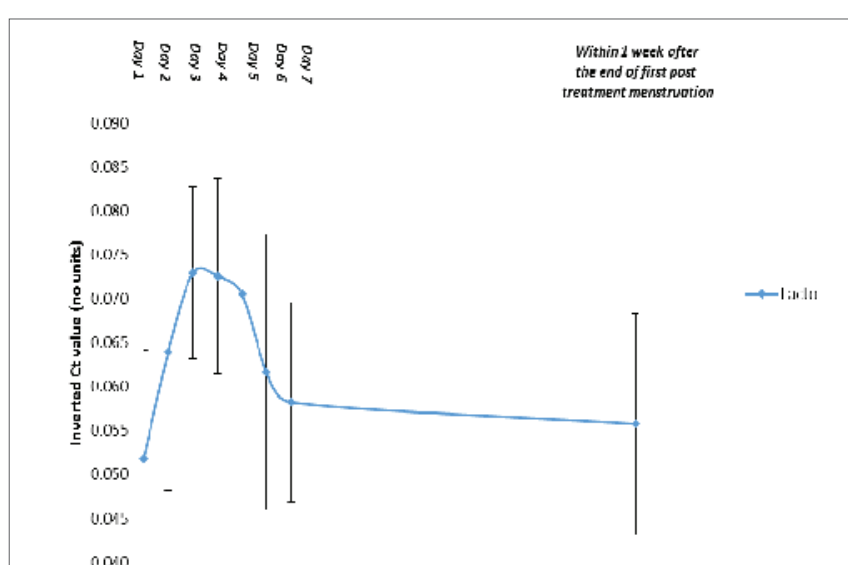

Figure 4: Quantification of Vaginal Lactobacilli during treatment: First 10 Patients

Data are shown as the mean and $95 \%$ confidence interval $(n=10)$. Abbreviations: $\mathrm{Ct}=$ Threshold cycle, Lacto=Lactobacilli

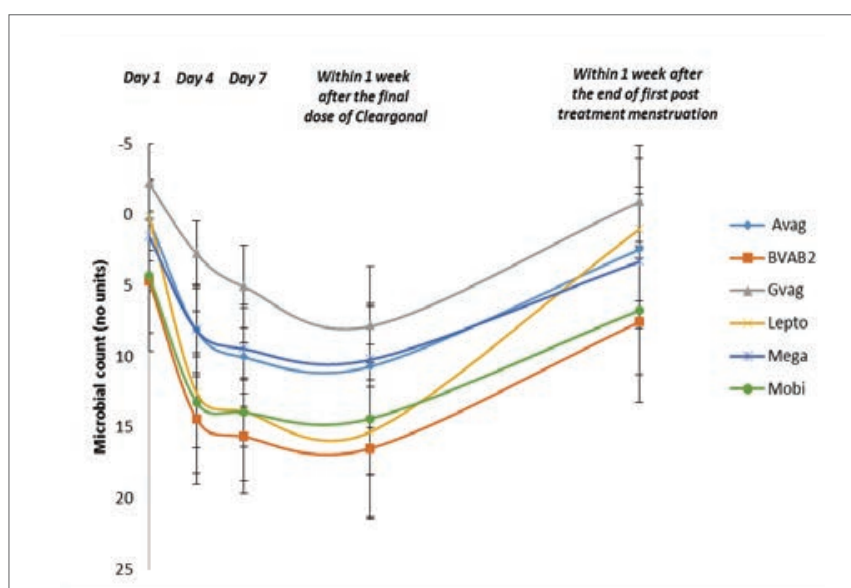

Figure 5: Quantification of Vaginal Pathogens: Subsequent 20 Patients. Data are shown as the mean and $95 \%$ confidence interval $(n=18)$.

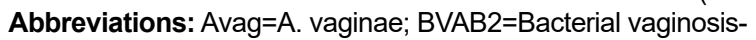
associated bacterium 2; Gvag=G. vaginalis, Lepto=Leptotrichia/ Sneathia spp.; Mega=Megasphaera spp.; Mobi=Mobiluncus spp.

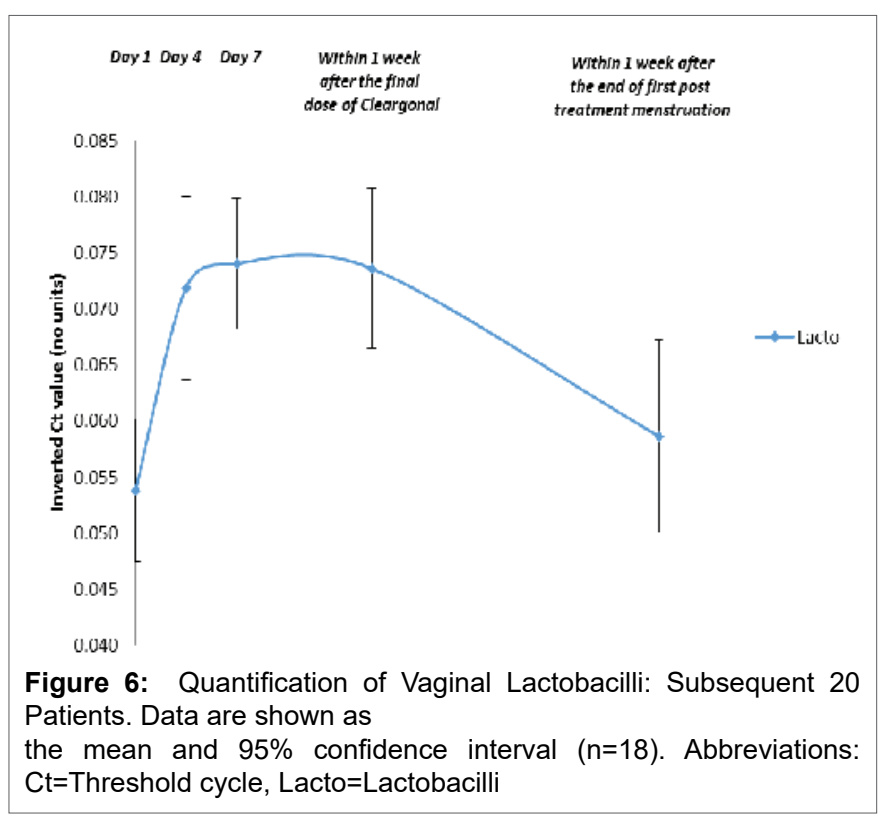




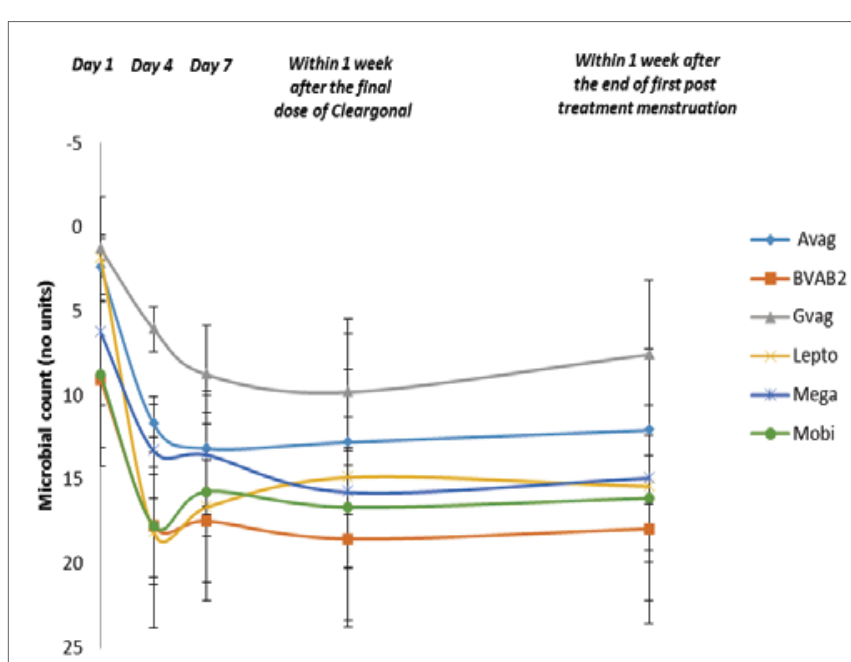

Figure 7: Quantification of Vaginal Pathogens: Subsequent 20 Patients (Patients with a Hay/Ison Grade of "Normal" or "Intermediate" within 1 Week after the End of the First Post- Treatment Menstruation) Data are shown as the mean and $95 \%$ confidence interval $(n=5)$. Abbreviations: Avag=A. vaginae, BVAB2=Bacterial vaginosisassociated bacterium 2, Gvag $=G$. vaginalis, Lepto=Leptotrichia/ Sneathia spp., Mega=Megasphaera spp., Mobi=Mobiluncus spp.

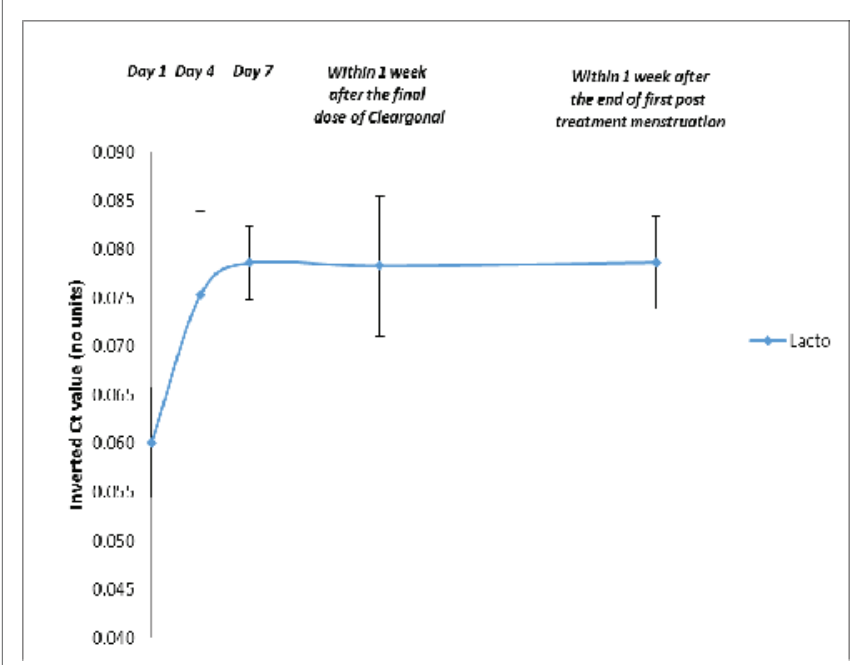

Figure 8: Quantification of Vaginal Lactobacilli: Subsequent 20 Patients (Patients with a

Hay/Ison Grade of "Normal" or "Intermediate" within 1 Week after the End of the First Post- Treatment Menstruation). Data are shown as the mean and $95 \%$ confidence interval $(n=5)$. Abbreviations: $\mathrm{Ct}=$ Threshold cycle, Lacto=Lactobacilli

Vaginal discharge improved in 17 patients (89.5\%) between Visit 1 and Visit 2, and was less severe at Visit 3 vs Visit 1 in 13 patients (76.5\%). Itch was an infrequent symptom at Visit 1 , but showed improvements at Visit 2 and Visit 3 in some patients. Pain was very infrequent, and no improvements were seen at Visit 2 or Visit 3 (Tables 5 and 6).

For the subsequent 20 patients, mean vaginal $\mathrm{pH}$ decreased from 5.3 at Visit 1, to 5.0 at day $4,4.8$ at day 7 and 4.6 for samples obtained within 1 week after the final dose of the novel product. For samples obtained within 1 week after the end of the first post- treatment menstruation, mean $\mathrm{pH}$ was 4.9 (Table 7 and Figures 9 and 10). $\mathrm{pH}$ for patients with a Hay/Ison grade of "Normal" or "Intermediate" within 1 week after the end of the first post-treatment menstruation are shown in Figure 11. The mean $\mathrm{pH}$ was maintained normal throughout the study period in these patients.

\section{Safety}

The product was well tolerated. No AEs judged to be related to the treatment were reported. There were no SAEs or deaths and no AEs resulted in a patient discontinuing study treatment or being withdrawn from the study. No unscheduled gynaecological examinations were needed during or after treatment.

\section{Adverse events}

There were 11 adverse events (AEs), all unique, in 10 patients see Table 8 . The most frequent AEs were Headache, 3 patients, (10\%), Fever, 2 patients, (7\%) and Cold 2 patients, (7\%). All other AEs occurred in a single patient. Two AEs, migraine and influenza, in 1 patient treated with a single dose of the novel product, patient 10 , were judged to be moderate while all other AEs were judged to be mild. All AEs were judged to be unrelated to the study treatment. No AE was judged to be serious. All AEs were resolved by the end of the study.

Table 4: Bacterial Vaginosis Symptoms at Visit 1, Visit 2 and Visit 3: First 10 Patients

\begin{tabular}{|c|c|c|c|c|}
\hline & Visit 1 & Visit 2 & Visit 3 \\
\hline & & Day 1 & $\begin{array}{c}1-2 \\
\text { weeks }\end{array}$ & $\begin{array}{l}\text { Within } 4 \text { weeks } \\
\text { after the first } \\
\text { post- treatment } \\
\text { menstruation }\end{array}$ \\
\hline \multirow{5}{*}{ Itch } & No. of observations & 10 & 10 & 10 \\
\hline & Severe, n (\%) & $0(0.0)$ & $0(0.0)$ & $0(0.0)$ \\
\hline & Moderate, $\mathrm{n}(\%)$ & $0(0.0)$ & $0(0.0)$ & $0(0.0)$ \\
\hline & Mild, n (\%) & $3(30.0)$ & $0(0.0)$ & $0(0.0)$ \\
\hline & No symptoms, n (\%) & $7(70.0)$ & $10(100.0)$ & $10(100.0)$ \\
\hline \multirow{5}{*}{ Pain } & No. of observations & 10 & 10 & 10 \\
\hline & Severe, n (\%) & $0(0.0)$ & $0(0.0)$ & $0(0.0)$ \\
\hline & Moderate, $\mathrm{n}(\%)$ & $0(0.0)$ & $0(0.0)$ & $0(0.0)$ \\
\hline & Mild, n (\%) & $1(10.0)$ & $1(10.0)$ & $0(0.0)$ \\
\hline & No symptoms, $\mathrm{n}(\%)$ & $9(90.0)$ & $9(90.0)$ & $10(100.0)$ \\
\hline \multirow{5}{*}{ Malodour } & No. of observations & 10 & 10 & 10 \\
\hline & Severe, $\mathrm{n}(\%)$ & $7(70.0)$ & $0(0.0)$ & $3(30.0)$ \\
\hline & Moderate, $\mathrm{n}(\%)$ & $3(30.0)$ & $2(20.0)$ & $4(40.0)$ \\
\hline & Mild, n (\%) & $0(0.0)$ & $3(30.0)$ & $0(0.0)$ \\
\hline & No symptoms, $\mathrm{n}(\%)$ & $0(0.0)$ & $5(50.0)$ & $3(30.0)$ \\
\hline \multirow{5}{*}{$\begin{array}{l}\text { Vaginal } \\
\text { discharge }\end{array}$} & No. of observations & 10 & 10 & 10 \\
\hline & Severe, n (\%) & $4(40.0)$ & $0(0.0)$ & $3(30.0)$ \\
\hline & Moderate, $\mathrm{n}(\%)$ & $2(20.0)$ & $2(20.0)$ & $3(30.0)$ \\
\hline & Mild, n (\%) & $3(30.0)$ & $4(40.0)$ & $0(0.0)$ \\
\hline & No symptoms, n (\%) & $1(10.0)$ & $4(40.0)$ & $4(40.0)$ \\
\hline
\end{tabular}


Table 5: Bacterial Vaginosis Symptoms at Visit 1, Visit 2 and Visit 3: Subsequent 20 Patients

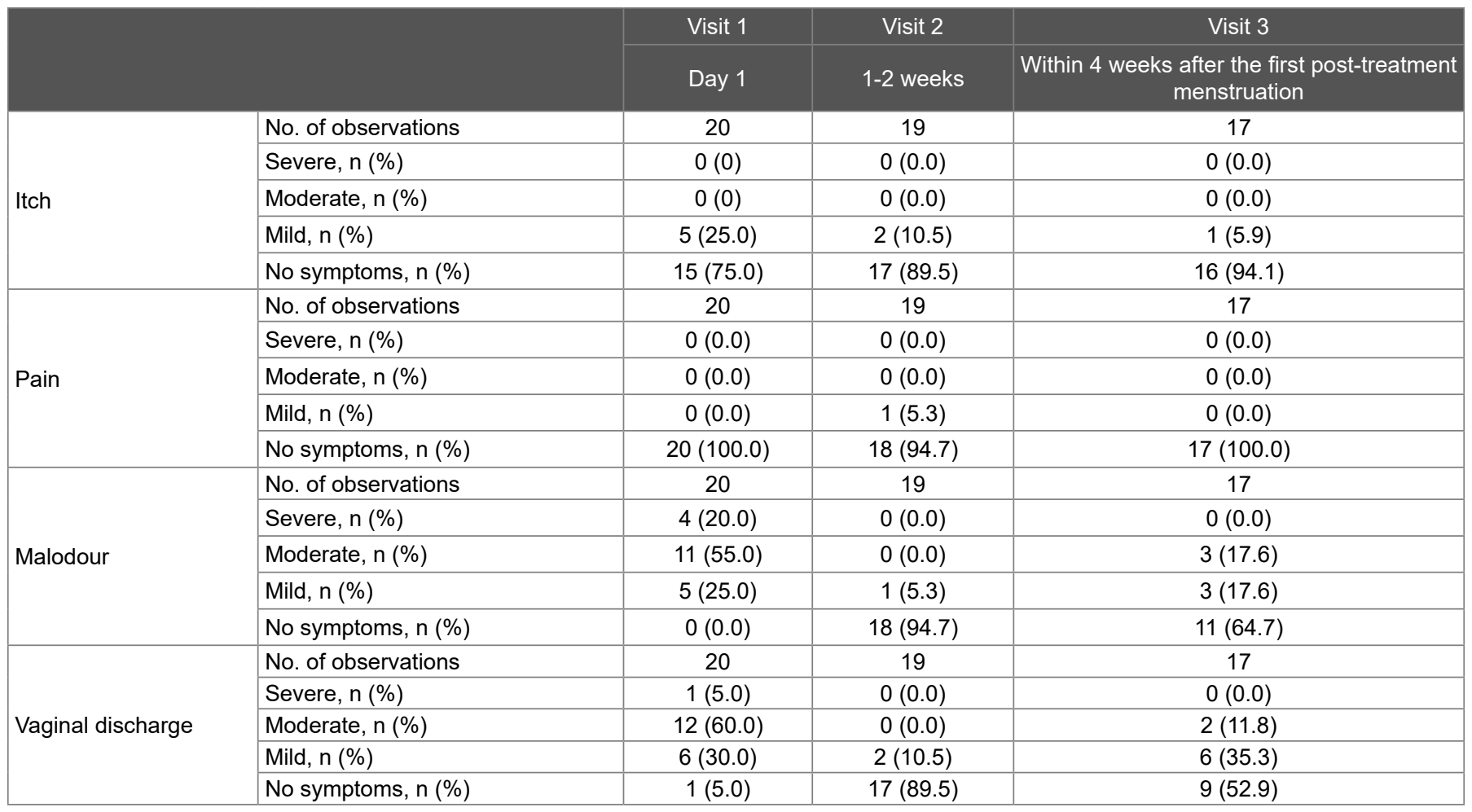

The decline in number of observations were caused by patients that discontinued the study prematurely.

Data for vaginal fluid pH are shown in Table 6 and Figure 9 (first 10 patients) and in Table 6 and Figure 10 (subsequent 20 patients). For the first 10 patients, mean vaginal pH decreased from 5.4 at Visit 1 (day 1 ) to 4.8 at day 2 and 4.5 at day 3 . Thereafter, it gradually increased to 4.9 at day 7 . For samples obtained within 1 week after the end of the first post-treatment menstruation, mean $\mathrm{pH}$ was 5.2 .

Table 6: Vaginal Fluid pH: First 10 Patients

\begin{tabular}{|c|c|c|c|c|c|c|c|c|c|}
\hline & \multirow{2}{*}{$\begin{array}{l}\text { Visit } 1 \\
\text { Day } 1\end{array}$} & \multicolumn{7}{|c|}{ Sampling } \\
\hline & & & Day 2 & Day 3 & Day 4 & Day 5 & Day 6 & Day 7 & $\begin{array}{l}\text { Within } 1 \text { week after the end of the first } \\
\text { post-treatment menstruation }\end{array}$ \\
\hline \multirow{8}{*}{$\mathrm{pH}$} & No. of observations & 10 & 10 & 10 & 8 & 8 & 8 & 8 & 7 \\
\hline & Mean & 5.4 & 4.8 & 4.5 & 4.6 & 4.7 & 4.7 & 4.9 & 5.2 \\
\hline & 95\% confidence interval & $5.2-5.5$ & $4.6-5.0$ & $4.2-4.8$ & $4.3-4.9$ & $4.4-5.0$ & $4.5-5.0$ & $4.6-5.2$ & $4.8-5.5$ \\
\hline & Min & 5.0 & 3.6 & 3.6 & 3.6 & 3.6 & 3.6 & 3.6 & 3.8 \\
\hline & Q1 & 5.1 & 4.7 & 4.1 & 4.3 & 4.6 & 4.6 & 5.0 & 5.0 \\
\hline & Median & 5.3 & 4.9 & 4.3 & 4.7 & 4.9 & 4.9 & 5.0 & 5.3 \\
\hline & Q3 & 5.6 & 5.2 & 5.0 & 4.8 & 5.1 & 5.1 & 5.1 & 5.5 \\
\hline & Max & 6.1 & 5.3 & 5.6 & 5.6 & 5.3 & 5.3 & 5.3 & 6.1 \\
\hline
\end{tabular}

Table 7: Vaginal Fluid pH: Subsequent 20 Patients

\begin{tabular}{|c|c|c|c|c|c|c|}
\hline & \multirow{2}{*}{$\begin{array}{l}\text { Visit } 1 \\
\text { Day } 1\end{array}$} & \multicolumn{2}{|c|}{ Treatment } & \multicolumn{2}{|r|}{ Sampling } \\
\hline & & & Day 4 & Day 7 & $\begin{array}{c}\text { Within } 1 \\
\text { week after the final dose }\end{array}$ & $\begin{array}{l}\text { Within } 1 \text { week after } \\
\text { the end of the first post-treatment menstruation }\end{array}$ \\
\hline \multirow{8}{*}{$\mathrm{pH}$} & No. of observations & 20 & 18 & 18 & 17 & 17 \\
\hline & Mean & 5.3 & 5.0 & 4.8 & 4.6 & 4.9 \\
\hline & 95\% confidence interval & $5.2-5.5$ & $4.7-5.3$ & $4.5-5.0$ & $4.3-4.9$ & $4.7-5.2$ \\
\hline & Min & 4.7 & 4.1 & 4.1 & 3.6 & 3.6 \\
\hline & Q1 & 5.3 & 4.7 & 4.1 & 4.1 & 4.7 \\
\hline & Median & 5.3 & 5.0 & 4.7 & 4.4 & 5.0 \\
\hline & Q3 & 5.6 & 5.3 & 5.2 & 5.0 & 5.3 \\
\hline & Max & 5.6 & 6.1 & 5.6 & 5.6 & 6.1 \\
\hline
\end{tabular}

Citation: Breding K, Lindal A, Sagna D, Larsson P-G (2018) Novel Non-Antibiotic Treatment of Bacterial Infections of the Vagina, A Proof of Concept Study using Single Dose and Triple Dose Regimen. Gynecol Women's Health Res 1(1): dx.doi.org/10.16966/2689-3096.108 
Table 8: Adverse Events by Primary Adverse Event Description

\begin{tabular}{|l|c|c|c|}
\hline \multicolumn{1}{|c|}{$\begin{array}{c}\text { Primary adverse event } \\
\text { description }\end{array}$} & $\begin{array}{c}\text { First 10 } \\
\text { patients }\end{array}$ & $\begin{array}{c}\text { Subsequent } 20 \\
\text { patients }\end{array}$ & All patients \\
\hline Fever & $1(10)$ & $1(5)$ & $2(7)$ \\
\hline Headache & $1(10)$ & $2(10)$ & $3(10)$ \\
\hline Migraine & $1(10)$ & & $1(3)$ \\
\hline Cold & $1(10)$ & $1(5)$ & $2(7)$ \\
\hline Influenza & $1(10)$ & & $1(3)$ \\
\hline Urethritis & & $1(5)$ & $1(3)$ \\
\hline Smarting before menstruation & & $1(5)$ & $1(3)$ \\
\hline
\end{tabular}

Data are shown as number of patients (\%)

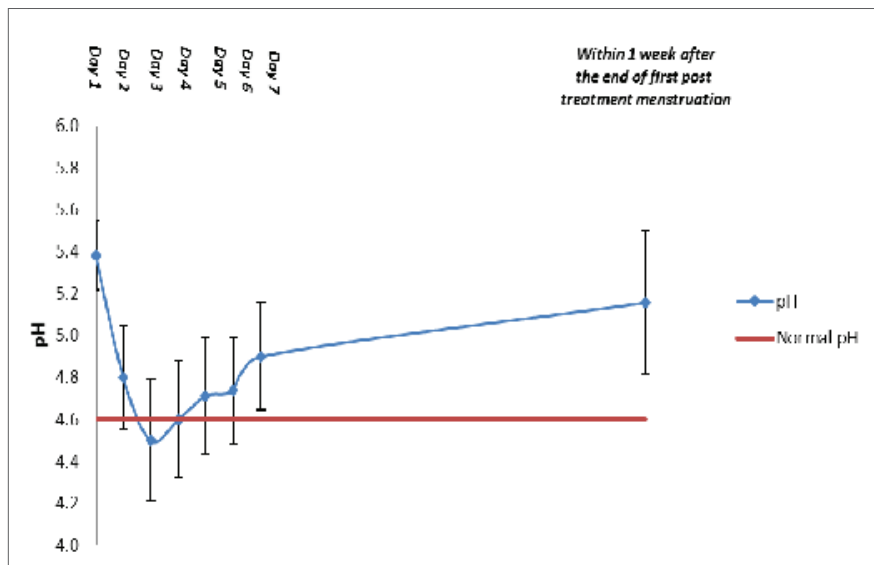

Figure 9: Vaginal Fluid pH: First 10 Patients. Data are shown as the mean and $95 \%$ confidence interval $(n=10)$.

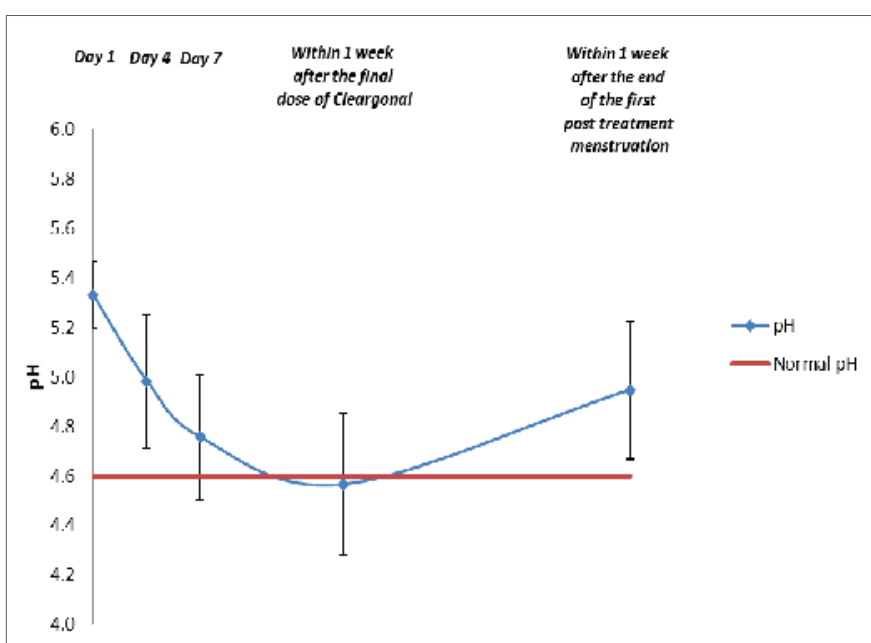

Figure 10: Vaginal Fluid pH: Subsequent 20 Patients. Data are shown as the mean and $95 \%$ confidence interval $(n=20)$.

\section{Discussion}

The results of the primary efficacy parameter were, for both groups, a rapid increase of healthy patients. After single treatment the effect, the number of patients defined as "normal" by Hay/Ison peaked day $2 / 3$ and after repeated dosing the peak came during treatment, day 1 to 7 . This is supported by the bacteriological data showing rapid onset for both treatment schedules and peaked (minimum pathogens

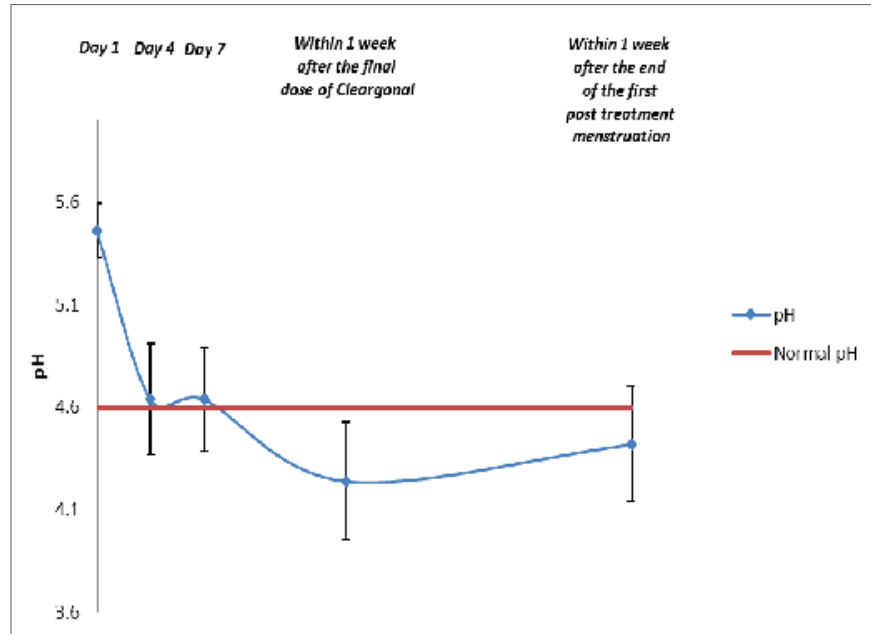

Figure 11: Vaginal Fluid pH: Subsequent 20 Patients. (Patients with a Hay/Ison Grade of "Normal" or "Intermediate" within 1 Week after the End of the First Post-Treatment Menstruation) Data are shown as the mean and $95 \%$ confidence interval $(n=5)$.

and maximum Lactobacilli) day 3 for single dose and day 1 to $7+$ for triple treatment. The results for therapeutic parameters, mainly $\mathrm{pH}$, malodour and abnormal discharge, support this statement. With the regard to duration it's interesting to note that the decline in effect demonstrated after single dose is much slower than if three doses are applied. One week after start of treatment the remaining cured patients were $12.5 \%$ while the number of improved were 50\% (normal + intermediary) after single dose. The corresponding Figures for triple doses are $50.0 \%$ and $83.3 \%$. This indicates an accumulation of the effect takes place when the number of doses is increased. This is further emphasized when therapeutic parameters such as malodour and vaginal discharge are considered. After the first post treatment menstruation in the single dose treated, $30 \%$ of the patients had no symptoms (malodour) compared to $64.7 \%$ after three doses. These results are superior to the symptom relief achieved with local antibiotic treatment [11].

As indicated by the PCR data on microbiology, the rapid reduction of pathogens coincides with a rapid increase in the number of Lactobacilli. This indicates that Lactobacilli species, L.crispatus, L.jenseni, L.gasseri, and L.iners are not sensitive to hydrogen peroxide. It should however not come as a surprise since Lactobacilli are known produce concentrations in the $\mathrm{mM}$ range of hydrogen peroxide in the surrounding media. Hydrogen peroxide and lactic acid producing Lactobacilli that colonise the vagina mucus have been reported to reduce the prevalence of bacterial vaginosis $[15,16]$. These $\mathrm{H}_{2} \mathrm{O}_{2}$-producing Lactobacilli were isolated from higher percentages of samples of vaginal fluid from healthy women than from those with bacterial vaginosis [17]. This bactericidal action seems inhibited by catalase but not by inactivated catalase and was not exhibited by non- $\mathrm{H}_{2} \mathrm{O}_{2}$-producing Lactobacilli. Vaginal peroxidase plus a halide (e.g., chloride) would be expected to enhance the lethal action of $\mathrm{H}_{2} \mathrm{O}_{2}$ formed by Lactobacilli, and this may 
contribute to the control of the vaginal flora [18]. As indicated earlier the antimicrobial activities of Lactobacilli strains against bacterial pathogens appears to be bifactual as Atassi et al. [19] have investigated the respective contribution of lactic acid and hydrogen peroxide in killing bacterial pathogen associated with human vagina with hydrogen peroxide concentration from $0-15 \mu \mathrm{g} / \mathrm{ml}$ of and the lactic acid concentration at $70 \mathrm{mM}$ showing a co-operative antimicrobial effect.

Themonoglycerideshavebeen used extensively in the cosmetic and food industry and are naturally occurring in sunflower oil. The antimicrobial properties of the monoglycerides have been shown in a double blind randomized study with 36 women how were treated with a $0.5 \%$ or $5 \%$ glycerol monolaurate gel. G. vaginalis and Candida was significantly reduced but Lactobacilli were not affected [20].

There seem to be a strong link between the parameters we have studied, clinical in the form of Hay/Ison, microbiological by $\mathrm{PCR}$ and therapeutic in the form of $\mathrm{pH}$, malodour, pain itch and vaginal discharge. One further indication on the strong correlation between clinical, therapeutic and bacteriological parameters was found when we compared bacteriology data from patients regarded as intermediary or healthy see Figure 7 and 8. In these patients there is no decrease in Lactobacilli and no increase in pathogens. This is very interesting that the pathogens still are possible to detect even if the women are free of BV. These findings are new and could in some way explain why women with BV have a high frequency of relapse. One theory of the relapse of BV is that the biofilm in vagina is maintained protecting the pathogenic bacteria [21].

Our result could suggest that treatment with this product will in some way even destroy the biofilm making the pathogenic bacteria more harmless.

In this initial study we have learned that the new formulation reduces pathogens and symptoms of infection rapidly with long term effect and this property may be useful for the treatment of other local vaginal infections such as STI. Another aspect that should be discussed is the statement that the components reducing $\mathrm{pH}$ and bacterial numbers, hydrogen peroxide and lactic acid, are unlikely to generate development of resistan ce. This is in strong contrast to the current preferred medication, Clindamycin, an antibiotic which might have a inhibiting effect on Lactobacilli and is subject to development of resistance [22-29].

One disadvantage of our study is that we did not treat or control her partner. In our study $75 \%$ patients had regular partners and we did not encourage the women to abstain from sexual intercourse nor to use condom.

\section{Conclusion}

Treatment of BV and symptoms thereof using a combination of hydrogen peroxide, lactic acid and a stabilising lipid, the novel formulation produced predictable results with a strong correlation between most clinical parameters, Hay/Ison and bacteriological ditto. The product had a rapid effect on Hay/ Ison grade and produced long-lasting improvements in symptoms, even in patients with relapse of BV. At three doses the cure rate, about $30 \%$ after post treatment menstruation, is in line with the results for frequent local vaginal metronidazole treatment but with superior symptoms relief. Increase of dose, dose frequency and number of doses will most likely increase cure rate. This statement is speculative since none comparative studies are made yet. The novel formulation did not interfere with Lactobacilli growth in contrast to local antibiotics such as clindamycine and was found safe and was tolerated by patients.

\section{Conflict of interest}

In order to conduct this study Skaraborgs Hospital in Skövde received scientific founding from Pharmiva $A B$, Sankt Lars väg 45, SE 22270 Sweden for salary to the scientific nurse.

\section{References}

1. Koumans EH, Sternberg M, Bruce C, McQuillan G, Kendrick J, et al. (2007) The prevalence of bacterial vaginosis in the United States, 2001-2004; associations with symptoms, sexual behaviors, and reproductive health. Sex Transm Dis 34: 864-869.

2. Martin HL, Richardson BA, Nyange PM, Lavreys L, Hillier SL, et al. (1999)Vaginal Lactobacilli, microbial flora, and risk of human immunodeficiency virus type 1 and sexually transmitted disease acquisition. J Infect Dis 180: 1863-1868.

3. Wiesenfeld HC, Hillier SL, Krohn MA, Landers DV, Sweet RL (2003) Bacterial vaginosis is a strong predictor of Neisseria gonorrhoeae and Chlamydia trachomatis infection. Clin Infect Dis 36: 663-668.

4. Brotman RM, Klebanoff MA, Nansel TR, Yu KF, Andrews WW, et al. (2010) Bacterial vaginosis assessed by gram stain and diminished colonization resistance to incident gonococcal, chlamydial, and trichomonal genital infection. J Infect Dis 202: 1907- 1915.

5. Nansel TR, Yu KF, Andrews WW, Zhang J, Klebanoff MA, et al. (2010) Bacterial vaginosis assessed by gram stain and diminished colonization resistance to incident gonococcal, chlamydial, and trichomonal genital infection. J Infect Dis 202:1907-1915.

6. Hillier SL, Nugent RP, Eschenbach DA, Krohn MA, Gibbs RS, et al. (1995) Association between bacterial vaginosis and preterm delivery of a low-birth-weight infant. The Vaginal Infections and Prematurity Study Group. N Engl J Med 333: 1737-1742.

7. Hay PE, Lamont RF, Taylor-Robinson D, Morgan DJ, Ison C, et al. (1994) Abnormal bacterial colonisation of the genital tract and subsequent preterm delivery and late miscarriage. BMJ 308: 295-298.

8. Leitich $\mathrm{H}$, Kiss $\mathrm{H}$ (2007) Asymptomatic bacterial vaginosis and intermediate flora as risk factors for adverse pregnancy outcome. Best Pract Res Clin Obstet Gynaecol 21: 375-390.

9. Shipitsyna E, Roos A, Datcu R, Hallén A, Fredlund H, et al. 2013 Composition of the vaginal microbiota in women of reproductive age--sensitive and specific molecular diagnosis of bacterial vaginosis is possible PLoS One 35: 525-532. 
10. Larsson PG, Brandsborg E, Forsum U, Pendharkar S, Andersen KK, et al. 2011 Extended antimicrobial treatment of bacterial vaginosis combined with human Lactobacilli to find the best treatment and minimize the risk of relapses. BMC Infect Dis 11: 223.

11. Chavoustie SE1, Jacobs M, Reisman HA, Waldbaum AS, Levy SF, et al. 2015 Metronidazole vaginal gel $1.3 \%$ in the treatment of bacterial vaginosis. J Low Genit Tract Dis 19: 129-134.

12. Schwebke JR, Marrazzo J, Beelen AP, Sobel JD (2015) A Phase 3, Multicenter, Randomized, Double-Blind, Vehicle- Controlled Study Evaluating the Safety and Efficacy of Metronidazole Vaginal Gel 1.3\% in the Treatment of Bacterial Vaginosis. Sex Transm Dis 42: 376-381.

13. Weissenbacher ER, Donders G, Unzeitig V, Martinez de Tejada B, Gerber $S$, et al. (2012) A comparison of dequalinium chloride vaginal tablets (Fluomizin ${ }^{\circledast}$ ) and clindamycin vaginal cream in the treatment of bacterial vaginosis: a single-blind, randomized clinical trial of efficacy and safety. Gynecol Obstet Invest 73: 8-15.

14. Larsson PG, Poutakidis G, Adolfsson A, Charonis G, Bauer P, et al. (2016) Treatment of Bacterial Vaginosis in Early Pregnancy and its Effect on Spontaneous Preterm Delivery and Preterm Premature Rupture of Membranes. Clin Microbiol 5: 259.

15. Wilks M, Wiggins R, Whiley A, Hennessy E, Warwick S, et al. (2004) Identification and $\mathrm{H}(2) \mathrm{O}(2)$ production of vaginal Lactobacilli from pregnant women at high risk of preterm birth and relation with outcome. J Clin Microbiol 42: 713-717.

16. Antonio MA, Rabe LK, Hillier SL (2005) Colonization of the rectum by Lactobacillus species and decreased risk of bacterial vaginosis. $J$ Infect Dis 192: 394-398.

17. D A Eschenbach, P R Davick, B L Williams, S J Klebanoff, K YoungSmith, et al. (1989) Prevalence of hydrogen peroxide-producing Lactobacillus species in normal women and women with bacterial vaginosis. J Clin Microbiol 27: 251-256.

18. Klebanoff SJ, Hillier SL, Eschenbach DA, Waltersdorph AM (1991) Control of the microbial flora of the vagina by $\mathrm{H} 2 \mathrm{O} 2$-generating Lactobacilli. J Infect Dis 164: 94-100.

19. Atassi F, Servin A (2010) Individual and co-operative roles of lactic acid and hydrogen peroxide in the killing activity of enteric strain
Lactobacillus johnsonii NCC933 and vaginal strain Lactobacillus gasseri KS120.1 against enteric, uropathogenic and vaginosisassociated pathogens.FEMS Microbiol Lett 304: 29-38.

20. Strandberg KL, Peterson ML, Lin YC, Pack MC, Chase DJ, et al. 2010 Glycerol monolaurate inhibits Candida and Gardnerella vaginalis in vitro and in vivo but not Lactobacillus. Antimicrob.Agents Chemother. 54: 597-601.

21. Swidsinski A, Mendling W, Loening-Baucke V, Ladhoff A, Swidsinski S, et al. 2005 Adherent biofilms in bacterial vaginosis. Obstet Gynecol. 106:1013-1023.

22. Boeke AJ, Dekker JH, van Eijk JT, Kostense PJ, Bezemer PD (1993) Effect of lactic acid suppositories compared with oral metronidazole and placebo in bacterial vaginosis: a randomised clinical trial. Genitourin Med. 69: 388-392.

23. Austin MN, Beigi RH, Meyn LA, Hillier SL (2005) Microbiologic response to treatment of bacterial vaginosis with topical clindamycin or metronidazole. J Clin Microbiol 43: 4492-4497.

24. Goldstein EJ, Citron DM, Cherubin CE, Hillier SL (1993) Comparative susceptibility of the Bacteroides fragilis group species and other anaerobic bacteria to meropenem, imipenem, piperacillin, cefoxitin, ampicillin/sulbactam, clindamycin and metronidazole. J Antimicrob Chemother 31: 363-372.

25. Goldstein EJ, Citron DM, Merriam CV, Warren YA, Tyrrell KL, et al. 2002 In vitro activities of Garenoxacin (BMS 84756) against 108 clinical isolates of Gardnerella vaginalis . Antimicrob Agents Chemother 46: 3995-3996.

26. Nagaraja P (2008) Antibiotic resistance of Gardnerella vaginalis in recurrent bacterial vaginosis. Indian J Med Microbiol 26: 155-157.

27. DeBacker E, Verhelst $R$, Verstraelen $H$, Claeys $G$, Verschraegen $G$, et al. 2006 Antibiotic susceptibility of Atopobium vaginae . BMC Infect Dis 6: 51.

28. Aroutcheva A, Simoes JA, Shott S, Faro S (2001) The inhibitory effect of clindamycin on Lactobacillus in vitro. Infect Dis Obstet Gynecol 9: 239-244.

29. Eriksson K, Larsson PG, Nilsson M, Forsum U (2011) Vaginal retention of locally administered clindamycin. APMIS 119: 373-376. 